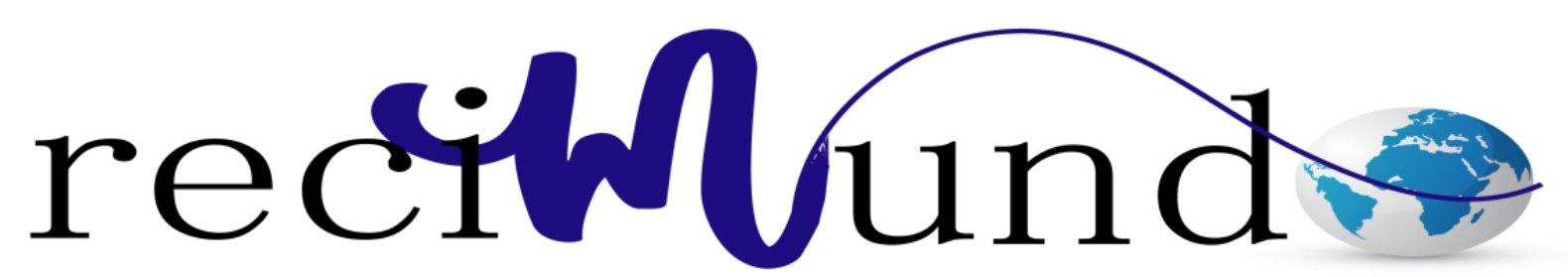

Revista Científica Mundo de la Investigación y el Conocimiento

\author{
Ana María Viteri Rojas a ; Karina Johana Cevallos Cárdenas ${ }^{\text {b; }}$ Migleth Natally \\ Cisneros López ${ }^{\mathrm{c}}$
}

Principios previos a la reanimación del recién nacido

Principles prior to resuscitation of the newborn

Revista Científica Mundo de la Investigación y el Conocimiento. Vol. 3 núm.3, septiembre, ISSN: 2588-073X, 2019, pp. 328-345

DOI: $10.26820 /$ recimundo/3.(3).septiembre.2019.328-345

URL: http://recimundo.com/index.php/es/article/view/524

Código UNESCO: 3205 Medicina Interna

Tipo de Investigación: Artículo de Revisión

Editorial Saberes del Conocimiento

Recibido: 15/05/2019

Aceptado: 23/06/2019

Publicado: 30/09/2019

Correspondencia: anyviteri031@ hotmail.com

a. Especialista en Cirugía General; Doctor en Medicina y Cirugía; Investigador Independiente; Guayaquil, Ecuador; anyviteri031@ @otmail.com

b. Magister en Atención Primaria y Clínica Infantil; Médico; Investigador Independiente; Guayaquil, Ecuador; karinacevallos75@hotmail.com

c. Médico; Investigador Independiente; Guayaquil, Ecuador; n_cisneros_1@ hotmail.com 


\section{Principios previos a la reanimación del recién nacido}

Vol. 3, núm. 3., (2019)

Ana María Viteri Rojas; Karina Johana Cevallos Cárdenas; Migleth Natally Cisneros López

\section{RESUMEN}

En la mayoría de los nacimientos, el recién nacido realiza con éxito la transición de la vida intrauterina hasta la extrauterina por sí sólo, no obstante, debido a los numerosos nacimientos que se producen a diario en todo el mundo, resulta importante el número de recién nacidos que necesitarán algún tipo de reanimación para realizar exitosamente esta transición. El objetivo de la presente investigación es plasmar los principios previos a la reanimación neonatal como lo son la anticipación a la reanimación (estudio de los factores de riesgo), personal y recursos necesarios. Así como la importancia de las guías científicas de reanimación del recién nacido. El diseño de investigación que se llevó a cabo es de tipo documental o bibliográfico. La anticipación se apoya fundamentalmente en la identificación de los factores de riesgo que aumenten la probabilidad de que el recién nacido requiera al momento de nacer de reanimación, y con esta información y el uso de las guías de práctica de las que se dispongan, organizar el personal y los insumos y equipos necesarios para llevar a cabo la ayuda que requiera en su transición el neonato. La consideración de los principios previos de la reanimación del recién nacido, con bases a las guías científicas de esta materia, en todo el mundo marca una pauta en la reducción de la mortalidad perinatal y las consecuencias negativas para la madre que esta pueda ocasionar.

Palabras Claves: Principios; Anticipación; Reanimación; Recién Nacido. 


\title{
Principios previos a la reanimación del recién nacido
}

Vol. 3, núm. 3., (2019)

Ana María Viteri Rojas; Karina Johana Cevallos Cárdenas; Migleth Natally Cisneros López

\begin{abstract}
In most births, the newborn successfully makes the transition from intrauterine to extrauterine life alone, however, due to the many births that occur daily worldwide, the number of newborns is important. born who will need some kind of resuscitation to successfully make this transition. The objective of this research is to capture the principles prior to neonatal resuscitation, such as the anticipation of resuscitation (study of risk factors), personnel and necessary resources. As well as the importance of the scientific guides of resuscitation of the newborn. The research design that was carried out is documentary or bibliographic. Anticipation is fundamentally based on the identification of risk factors that increase the likelihood that the newborn will require at the time of birth resuscitation, and with this information and the use of the available practice guides, organize the personnel and the necessary supplies and equipment to carry out the help that the newborn needs in its transition. The consideration of the previous principles of resuscitation of the newborn, based on the scientific guidelines of this matter, worldwide marks a pattern in the reduction of perinatal mortality and the negative consequences for the mother that this may cause.
\end{abstract}

Key words: Principles; Anticipation; Resuscitation; Newborn Born. 


\section{Principios previos a la reanimación del recién nacido}

Vol. 3, núm. 3., (2019)

Ana María Viteri Rojas; Karina Johana Cevallos Cárdenas; Migleth Natally Cisneros López

\section{Introducción.}

En la mayoría de los nacimientos, el recién nacido realiza con éxito la transición de la vida intrauterina hasta la extrauterina por sí sólo, no obstante, debido a los numerosos nacimientos que se producen a diario en todo el mundo, resulta importante el número de recién nacidos que necesitarán alguna ayuda para realizar exitosamente esta transición.

Es fundamental que la atención en la sala de partos hospitalario cuente con un equipo debidamente entrenado en la reanimación neonatal a los fines de ayudar al recién nacido en la etapa de transición y tratar las complicaciones que se puedan generar. La reducción de morbilidad y morbimortalidad neonatales es el objetivo primordial de la atención en la sala de partos enfocada en la prevención de las complicaciones.

“La mayor parte de las defunciones de recién nacidos $(75 \%)$ ocurren durante la primera semana de vida, y aproximadamente 1 millón de recién nacidos mueren en las 24 primeras horas de vida". Una de las principales complicaciones relacionadas con el parto es la asfixia perinatal, la cual se encuentra asociada a la mayor parte de las defunciones de recién nacidos en 2016. (Organización Mundial de la Salud, 2019)

En tal sentido, Jiménez Pérez \& García Villanueva (2017) acotan que cada año nacen en el mundo más de 130 millones de niños, de dicha cantidad un 10\% aproximadamente, requiere de alguna forma de intervención inmediata después del nacimiento. Asimismo, se tiene que cifras estimadas muestran unos 4 millones de muertes neonatales en todo el mundo, de las cuales el $25 \%$ corresponden a la causa secundaria de asfixia perinatal. "La asfixia perinatal es la causa principal que condiciona la necesidad de reanimación, aproximadamente un $6 \%$ de los recién nacidos, 


\section{Principios previos a la reanimación del recién nacido}

Vol. 3, núm. 3., (2019)

Ana María Viteri Rojas; Karina Johana Cevallos Cárdenas; Migleth Natally Cisneros López

porcentaje que se eleva hasta alcanzar el $80 \%$ en los niños prematuros, precisan reanimación en los minutos inmediatos al nacimiento". (p. 31)

La Secretaría Distrital de Salud de Bogotá (2015) en su Guía Clínica de lineamiento técnico para el manejo de la asfixia perinatal, define la asfixia perinatal como "la condición en la que se presenta una alteración en el suministro de oxígeno al momento del nacimiento, que surge como consecuencia de diferentes noxas, durante el trabajo de parto, el expulsivo o los primeros minutos posteriores al nacimiento". (p. 9)

Parte importante del éxito de la reanimación neonatal va a depender de la anticipación a la misma, esto es, la preparación que se realice antes de que se produzca el parto que contiene preparación del equipo que va a asistir el parto, estudio de los factores de riesgo y de la condición hemodinámica que presenta el feto durante el trabajo de parto. Asimismo, resulta imperioso el uso de los protocolos de atención basados en las guías científicas para la reanimación del recién nacido reconocidas y usadas a nivel mundial.

El objetivo de la presente investigación es plasmar los principios previos a la reanimación neonatal como lo son la anticipación a la reanimación (estudio de los factores de riesgo), personal y recursos necesarios. Así como la importancia de las guías científicas de reanimación del recién nacido.

\section{Materiales y Métodos.}

Para llevar a cabo la presente investigación, en primer lugar, se procedió a identificar aquellos documentos que estaban acordes con los objetivos planteados para este estudio. Tales 


\section{Principios previos a la reanimación del recién nacido}

Vol. 3, núm. 3., (2019)

Ana María Viteri Rojas; Karina Johana Cevallos Cárdenas; Migleth Natally Cisneros López documentos fueron buscados en las principales bases de datos electrónicas relacionadas con las ciencias de la salud, de manera general y más específicamente relacionadas con las áreas de obstetricia, cardiología y enfermería: Mayo Clinic, MedlinePlus, Organización Mundial de la Salud, Organización Panamericana de la Salud, sciELO, ELSEVIER, Infomed, Asociación Americana del Corazón y Asociación Americana de Pediatría.

La investigación se limitó a la búsqueda y revisión sistemática de material bibliográfico, con la finalidad de sintetizar la evidencia encontrada. En este orden de ideas, la presente investigación se enmarca dentro del tipo documental bibliográfico.

Es importante destacar que la búsqueda se llevó a cabo por medio del uso de los siguientes descriptores y calificadores: "reanimación del recién nacido", "factores de riesgo de la asfixia perinatal", "asfixia perinatal", "reanimación neonatal" y "guías científicas de reanimación neonatal". El material obtenido se filtró usando criterios de relevancia, actualidad, idioma español e inglés y correlación temática. Por último, se destaca que la fecha de publicación se ubicó en los últimos cinco años para garantizar el principio de actualidad de acuerdo con la temática. No se descartó el tipo de material bibliográfico.

\section{Resultados.}

Principios previos a la reanimación neonatal. Preparación, anticipación y recursos necesarios

Anticipación a la necesidad de reanimación del recién nacido.

La anticipación o preparación para la reanimación del recién nacido es fundamental en cada parto. Dentro de los elementos principales de la preparación se encuentran el estudio de los factores 


\section{Principios previos a la reanimación del recién nacido}

Vol. 3, núm. 3., (2019)

Ana María Viteri Rojas; Karina Johana Cevallos Cárdenas; Migleth Natally Cisneros López

de riesgo que alertan la necesidad de la transición o reanimación del recién nacido. Una correcta identificación y consideración de estos factores de riesgo ayudan en la toma de decisiones para la preparación correcta del personal necesario para atender el parto.

Sánchez López (2016) se refieren a la anticipación a la reanimación neonatal como aquella donde se debe considerar, entre sus principales elementos, la evaluación del riesgo perinatal, acompañado de un sistema organizador de personal basado en dicho riesgo y un método organizado con la finalidad de garantizar el debido acceso al material y equipo de reanimación.

\section{Equipo perinatal.}

Es imperioso que por lo menos exista una persona que pueda realizar los pasos iniciales de reanimación neonatal en cada parto, cuya responsabilidad única sea la atención del recién nacido. Igualmente, es importante que ante la presencia de factores de riesgo perinatal que aumenten el requerimiento de reanimación, esté disponible inmediatamente, personal adicional que posea las habilidades en reanimación, esto es: “compresiones torácicas, intubación orotraqueal y colocación de catéter umbilical".

El Área de Trabajo de Reanimación Neonatal - Comité d6e Estudios Feto-neonatales CEFEN de Argentina (2018) acerca del equipo perinatal destacan el deber que tienen las personas que lo conforman, de reunirse antes del nacimiento, por cuanto el asesoramiento prenatal resulta vital para la toma de decisiones, sobre todo en aquellos partes de alto riesgo. "Es clave una adecuada comunicación entre el equipo obstétrico y neonatal. El equipo encargado de la recepción del RN debe revisar la situación clínica y definir un plan de acción”. Es imperiosa la evaluación 


\section{Principios previos a la reanimación del recién nacido}

Vol. 3, núm. 3., (2019)

Ana María Viteri Rojas; Karina Johana Cevallos Cárdenas; Migleth Natally Cisneros López

de los factores de riesgo, así como identificar un líder de equipo, delegar tareas específicas, bien definidas y designar un encargado de documentar todo lo que acontezca. (p. S62)

Factores de riesgo que pueden ayudar a predecir qué bebés necesitarán reanimación.

Este elemento básico en la anticipación a la reanimación es sumamente importante para identificar aquellos partos que tienen mayor probabilidad de necesitar reanimación, sin menoscabo de que estos factores no se presenten, ya que siempre se debe estar preparado para brindar el apoyo necesario al recién nacido en su transición desde adentro del útero a estar fuera de este.

Si bien es cierto que, en la mayoría de los casos, la necesidad de reanimación o estabilización resulta predecible, tampoco es menos cierto que no siempre es así. La identificación de estos factores resulta importante debido a que la mayoría de los recién nacidos que la requieren al momento del parto pueden también ser identificados.

En este orden de ideas, Llambías et al. (2016) consideran que el adecuado manejo de las pacientes que presentan factores de riesgo relacionados con la asfixia perinatal facilita un correcto manejo preventivo y terapéutico de las consecuencias negativas que se puedan presentar, las cuales pueden afectar tanto a la madre como al feto. "La identificación de los factores de riesgo más frecuentes y susceptibles de modificación permite adecuar la atención perinatológica y neonatológica a las condiciones concretas de riesgo del binomio madre-hijo”. (p. 31)

Rivera, Lara, \& Baró (2017) dividen las causas de la asfixia perinatal o factores de riesgo en factores que provienen de la madre, obstétricos, úteros placentarios y por último los asociados propiamente con el feto, los cuales se mencionan en las siguientes listas: 


\section{Principios previos a la reanimación del recién nacido}

Vol. 3, núm. 3., (2019)

Ana María Viteri Rojas; Karina Johana Cevallos Cárdenas; Migleth Natally Cisneros López

Factores maternos

- Hemorragia en el tercer trimestre.

- Infecciones (urinarias, corioamnionitis, sepsis).

- Hipertensión inducida por el embarazo.

- Hipertensión crónica.

- Anemia.

- Colagenopatías.

- Intoxicación por drogas.

- Comorbilidad obstétrica.

Obstétricos

- Líquido amniótico meconial

- Incompatibilidad céfalo-pélvico

- Uso de medicamentos

- Presentación fetal anormal

- Trabajo de parto prolongado o anormal

- Parto instrumentado o cesárea

- Ruptura prematura de membrana

- Oligoamnioso polihidramnios. 


\section{Principios previos a la reanimación del recién nacido}

Vol. 3, núm. 3., (2019)

Ana María Viteri Rojas; Karina Johana Cevallos Cárdenas; Migleth Natally Cisneros López

Útero-placentarias

- Anormalidades de cordón: Circular del cordón irreductible, procúbito, prolapso del cordón umbilical.

- Anormalidades placentarias: desprendimiento prematuro de placenta, placenta previa, alteraciones de la contractilidad uterina, hipertonía uterina, hipotonía uterina.

- Anormalidades uterinas anatómicas: Útero bicorne.

Fetales

- Alteraciones de la frecuencia cardíaca fetal: Bradicardia, taquicardia, arritmia.

- Percepción de disminución de movimientos fetales por la madre

- Retardo del crecimiento intrauterino

- Prematuridad

- Bajo peso

- Macrosomía fetal

- Postmadurez

- Malformaciones congénitas

- Eritroblastosis fetal

- Fetos múltiples

- Retraso en el crecimiento intrauterino. 


\section{Principios previos a la reanimación del recién nacido}

Vol. 3, núm. 3., (2019)

Ana María Viteri Rojas; Karina Johana Cevallos Cárdenas; Migleth Natally Cisneros López

Personal.

La Sociedad Española de Neonatología afirma que en las salas de partos debe contar con una persona debidamente formada en reanimación neonatal inicial la cual debe estar encargada únicamente del recién nacido, y es fundamental que la persona capaz de llevar a cabo la reanimación completa, se ubique con facilidad en caso de ser requerida. Con respecto al número de personal, se tiene que, ante situaciones de compromiso fetal severo, es imperioso que sean dos las personas disponibles para realizar una reanimación neonatal completa, en cuyo caso una de estas llevará a cabo la ventilación y la otra se encargará de monitorizar la frecuencia cardiaca o practicar el masaje cardíaco en caso de requerirse. "En el caso que el recién nacido precisara de medicación sería recomendable una tercera persona encargada de su preparación y administración, por eso es indispensable que las unidades de intensivos neonatales estén anexas a la sala de paritorios”. (Jiménez Pérez \& García Villanueva, 2017, p. 39)

\section{Equipos y Suministros.}

La Academia Americana de Pediatría \& Asociación Americana del Corazón (2016) por medio de su Programa de Reanimación Neonatal (PRN) ha sido de gran ayuda para los proveedores de atención médica, específicamente para adquirir el conocimiento y las destrezas que les permite realizar la reanimación neonatal. En este programa se recomienda que para la reanimación se debe disponer de los siguientes recursos necesarios:

Equipo de succión.

- Pera de goma 


\section{Principios previos a la reanimación del recién nacido}

Vol. 3, núm. 3., (2019)

Ana María Viteri Rojas; Karina Johana Cevallos Cárdenas; Migleth Natally Cisneros López

- Succión mecánica y tubos

- Catéteres de succión, 5F o 6F, 10F, 12F o 14F

- Sonda de alimentación $8 \mathrm{~F}$ y jeringa grande

- Aspirador de meconio

Equipo de ventilación por presión positiva.

- Dispositivo para proporcionar ventilación por presión positiva

- Máscaras, tamaños para recién nacidos y bebés prematuros

- Fuente de oxígeno

- Fuente de aire comprimido

- Mezclador de oxígeno para mezclar oxígeno y aire comprimido con un

- Medidor de flujo (velocidad de flujo configurada en $101 / \mathrm{min}$ ) y tubos

- Oxímetro de pulso con sensor y tapa

- Tabla de objetivo de saturación de oxígeno

Equipo de intubación.

- Laringoscopio de hojas rectas, $\mathrm{N} .^{\circ} 0$ (prematuro) y $\mathrm{N} .^{\circ} 1$ (a término)

- Focos y baterías adicionales para el laringoscopio

- Tubos endotraqueales, 2.5,3.0,3.5 mm de diámetro interno (DI)

- Estilete (opcional)

- Cinta métrica

- Tabla de profundidad de inserción de tubo endotraqueal 


\section{Principios previos a la reanimación del recién nacido}

Vol. 3, núm. 3., (2019)

Ana María Viteri Rojas; Karina Johana Cevallos Cárdenas; Migleth Natally Cisneros López

- Tijeras

- Cinta adhesiva a prueba de agua o dispositivo para asegurar el tubo

- Toallitas con alcohol

- Detector de CO 2 o capnógrafo

- Máscara laríngea (o dispositivo supraglótico similar) y jeringa de 5 mi

- Sonda orogástrica de 5F o 6F si la máscara laríngea presenta puerto de

- Inserción

Medicamentos.

- Adrenalina 1:10 $000(0.1 \mathrm{mg} / \mathrm{ml})$, ampollas de $3 \mathrm{mi} \mathrm{o} 10 \mathrm{mi}$

- Solución salina normal para expansión de volumen de 100 o 250 mi

- Dextrosa al $10 \%, 250 \mathrm{mi}$ (opcional)

- Solución salina normal para enjuagues

- Jeringas (1 mi, $3 \mathrm{mi}$ o $5 \mathrm{mi}, 20$ a $60 \mathrm{mi}$ ). (p. 26)

Suministros para cateterización de vaso umbilical.

- Guantes estériles

- Solución de preparación antiséptica

- Cinta umbilical

- Pinza pequeña (hemostática)

- Fórceps (opcional)

- Escalpelo 


\section{Principios previos a la reanimación del recién nacido}

Vol. 3, núm. 3., (2019)

Ana María Viteri Rojas; Karina Johana Cevallos Cárdenas; Migleth Natally Cisneros López

- Catéteres umbilicales (lumen simple), 3.5F o 5F

- Llave de paso de tres vías

- Jeringas (3-5 mi)

- Agujas o dispositivo de punción para sistema sin aguja

- Solución salina normal para enjuagues

- Venda adhesiva transparente para sujetar temporalmente el catéter venoso

- Umbilical al abdomen (opcional)

Varios.

- Guantes y protección personal adecuados

- Calentador radiante u otra fuente de calor

- Sensor de temperatura con tapa de sensor para el calentador radiante (para utilizar durante una reanimación prolongada)

- Superficie de reanimación firme y acolchonada

- Cronómetro/reloj con segundero

- Mantas

- Gorro

- Estetoscopio (con cabeza neonatal)

- Cinta adhesiva, $1 / 2$ o 3/4 pulgadas

- Electrodos del monitor cardíaco electrónico (ECG) y monitor ECG

- Aguja intraósea (opcional) 


\section{Principios previos a la reanimación del recién nacido}

Vol. 3, núm. 3., (2019)

Ana María Viteri Rojas; Karina Johana Cevallos Cárdenas; Migleth Natally Cisneros López

En casos de bebés muy prematuros.

- Hoja de laringoscopio tamaño 00 (opcional)

- Bolsa plástica de grado alimenticio (1 galón de volumen) o envoltorio

- plástico

- Colchón térmico

- Incubadora portátil para mantener la temperatura del bebé mientras se lo traslada a la guardería

Principales guías científicas para la reanimación del recién nacido

La American Heart Association (AHA) es la encargada de publicar las Guías científicas para Reanimación Cardiopulmonar (RCP) y Atención Cardiovascular de Emergencia (ACE), “que conforman la base de los protocolos que salvan vidas usados por profesionales de la salud, empresas y hospitales en los Estados Unidos y en todo el mundo". La AHA trabaja mancomunadamente con organizaciones de la salud y del corazón, las cuales se destacan por ser líderes a nivel mundial en la recopilación y revisión de avances científicos, específicamente en materia de reanimación. Asimismo, este organismo es el responsable de publicar las Guías de la AHA para RCP y ACE, las cuales sirven como base para otras guías y productos que vitalizan a la ciencia para salvar vidas. (American Heart Association, 2019)

Arnett, et al. (2019) mencionan en su trabajo que por cuanto la última guía de reanimación cardiopulmonar de la AHA es de 2015, a partir del año 2017 se han implementado y se siguen implementando una serie de modificaciones a las pautas con la finalidad de mejorarla, acortando las pautas y haciendo que su uso sea más fácil y sencillo. Estas pautas son escritas y presentadas 


\section{Principios previos a la reanimación del recién nacido}

Vol. 3, núm. 3., (2019)

Ana María Viteri Rojas; Karina Johana Cevallos Cárdenas; Migleth Natally Cisneros López

"en un formato de fragmento de conocimiento modular, en el que cada fragmento incluye una tabla de recomendaciones, una breve sinopsis, texto de apoyo para recomendaciones específicas y, cuando sea apropiado, diagramas de flujo o tablas adicionales". (p. 1378)

\section{Conclusiones.}

Es importante identificar de forma precoz los factores de riesgo de aumentan las probabilidades de que exista asfixia neonatal o alguna complicación durante el parto y se requiera reanimar al recién nacido, por cuanto representa un elemento de anticipación que permite preparar y tomar las medidas necesarias para atender el parto de alto riesgo.

En este orden de idea ante la detección de estos factores de riesgo resulta fundamental la conformación de un equipo debidamente entrenado en reanimación neonatal, capaz de tomar las decisiones adecuadas y prestar el mejor servicio tanto al recién nacido como a la madre.

Por otra parte, es necesario que el centro de salud en su sala de parto cuente con el equipo necesario para brindar la debida atención al recién nacido y la madre.

La consideración de los principios previos de la reanimación del recién nacido, con bases a las guías científicas de esta materia, en todo el mundo marca una pauta en la reducción de la mortalidad perinatal y las consecuencias negativas para la madre que esta pueda ocasionar. 


\section{Principios previos a la reanimación del recién nacido}

Vol. 3, núm. 3., (2019)

Ana María Viteri Rojas; Karina Johana Cevallos Cárdenas; Migleth Natally Cisneros López

\section{Referencias Bibliográficas}

Academia Americana de Pediatría \& Asociación Americana del Corazón. (2016). Reanimación Neonatal (7 ed.). (G. M. Weiner, Ed.) Northwest Point. Recuperado el 19 de septiembre de 2019 , https://www.hbint.org/uploads/8/4/8/2/84824300/reanimacion_neonatal_7a_edicion_pdf. pdf

American Heart Association. (2019). international.heart.org. Recuperado el 20 de septiembre de 2019, de https://international.heart.org/es

Área de Trabajo de Reanimación Neonatal - Comité de Estudios Feto-neonatales CEFEN. (2018). Actualización en reanimación cardiopulmonar neonatal. Arch Argent Pediatr, 116(3), S59S70. Recuperado el 17 de septiembre de 2019, de https://www.sap.org.ar/docs/publicaciones/archivosarg/2018/v116n3a32s.pdf

Arnett, D. K., Blumenthal, R., Albert, M., Buroker, A. B., Goldberger, Z. D., Hahn, E. J., . . . Smith, S. (2019). 2019 ACC/AHA Guideline on the Primary Prevention of Cardiovascular Disease: Executive Summary. Journal of the American College of Cardiology, 74(10), 1376-1414. Recuperado el 20 de septiembre de 2019, de http://www.onlinejacc.org/content/74/10/1376

Jiménez Pérez, J. M., \& García Villanueva, S. (2017). Intervención estandarizada en la reanimación. Revista Enfermería C y R, 9(2), 29-45. Recuperado el 19 de septiembre de 2019, http://www.revistaenfermeriacyl.com/index.php/revistaenfermeriacyl/article/viewFile/19 $8 / 172$

Llambías, A., Reyes, W., Pérez, R., Carmenate, L., Pérez, L., \& Díaz, G. (2016). Factores de riesgo de la asfixia perinatal. Mediciego, 22(4), 30-35. Recuperado el 20 de septiembre de 2019, de https://www.medigraphic.com/pdfs/mediciego/mdc-2016/mdcs161d.pdf

Organización Mundial de la Salud. (19 de septiembre de 2019). Organización Mundial de la Salud. Recuperado el 20 de septiembre de 2019, de https://www.who.int/es/news-room/factsheets/detail/newborns-reducing-mortality

Rivera, M. A., Lara, N. T., \& Baró, T. (2017). Asfixia al nacer: factores de riesgo materno y su repercusión en la mortalidad neonatal. Revista de Información Científica, 96(6), 11431152. Recuperado el 20 de septiembre de 2019, de http://www.revinfcientifica.sld.cu/index.php/ric/article/view/1794/3382

Sánchez López, H. (10 de agosto de 2016). Sociedad Interamericana de Cardiología. Recuperado $\begin{array}{llllll}\text { el } & 20 & \text { de } & \text { septiembre } & \text { de } & 2019,\end{array}$ http://www.siacardio.com/consejos/pedriatia/actualizacion-2015-de-las-guias-de-la- 


\section{Principios previos a la reanimación del recién nacido}

Vol. 3, núm. 3., (2019)

Ana María Viteri Rojas; Karina Johana Cevallos Cárdenas; Migleth Natally Cisneros López

academia-americana-del-corazon-aha-para-la-reanimacion-cardiopulmonar-y-la-atencionde-las-urgencias-cardiovasculares/

Secretaría Distrital de Salud de Bogotá. (2015). Lineamiento técnico para el manejo de la asfixia perinatal. Guía, Bogotá. Recuperado el 20 de septiembre de 2019, de http://www.saludcapital.gov.co/DDS/Guas\%20de\%20Asfixia\%20Perinatal/Gu\%C3\%AD a\%20de\%20Asfixia\%20Perinatal.pdf 